
\title{
28 Research Suare \\ LncRNA TCL6 Contributes to CSC-like Properties via Modulating TP53 in HCC
}

\section{Lihua Luo}

Wuhan University Enshi Clinical College: Central Hospital of Enshi Tujia and Miao Autonomous Prefecture

\section{Yong Zhou}

Wuhan University Enshi Clinical College: Central Hospital of Enshi Tujia and Miao Autonomous

Prefecture

\section{Min Jin}

Huazhong University of Science and Technology Tongji Medical College

\section{Bai Li}

Second Military Medical University First Hospital: Changhai Hospital

\section{Tao Zhang}

Huazhong University of Science and Technology Tongji Medical College

\section{Jia-yao Zhang}

Wuhan University Enshi Clinical College: Central Hospital of Enshi Tujia and Miao Autonomous

Prefecture

\section{Xiang Donghua}

Wuhan University Enshi Clinical College: Central Hospital of Enshi Tujia and Miao Autonomous

Prefecture

Jin-mao Li ( $D$ lijinmao@sina.com.cn )

Enshi Clinical College of Wuhan University

\section{Research}

Keywords: Hepatocellular carcinoma, inhibit, TCL6, liver cancer stem cells

Posted Date: September 13th, 2021

DOI: https://doi.org/10.21203/rs.3.rs-850413/v1

License: (9) This work is licensed under a Creative Commons Attribution 4.0 International License.

Read Full License 


\section{Abstract}

\section{Background}

Hepatocellular carcinoma (HCC) is the most common malignant tumors, accounting for most of the adult primary liver cancer. Herein, we aimed to analyze the expression of long non-coding RNA-T cell leukemia/lymphoma 6 (IncRNA-TCL6) in HCC and elucidate its mechanism involved in the HCC progression.

Methods

e performed RNA extraction and quantitative real-time polymerase chain reaction assays, spheroid formation assays, flow cytometry and western blot assays to assess the effect of TCL6 on the liver CSCs marker CD133 expression rate, sphere-forming ability of liver stem cells, and the relationship between TCL6 expression and stem cell factor (TP53, P21, CD44, KLF4, OCT4, Nanog, and Sox2). In addition, we used a dual luciferase assay to verify the relationship between miR-106a-5p and TP53.

Results

Knockdown of TCL6 expression significantly improved the CD133 expression rate and the liver stem cells sphere-forming ability in HCC, while TCL6 overexpression in HCC showed the opposite effect. Knockdown of TCL6 upregulated the KLF4mRNA expression, while TCL6 overexpression in HCC inhibited the TP53 and CDKN1A expression. Western blot assays showed that TCL6 expression was positively correlated with TP53 and P21, while negatively correlated with stem cell factor. Dual luciferase assay showed that TP53 was a target of miR-106a-5p.

Conclusion

Results suggested that reprogramming-related TCL6 may be a novel tumor suppressor gene in HCC, which inhibits the self-renewal of liver CSCs, in part by promoting the TP53 expression.

\section{Introduction}

Hepatocellular carcinoma (HCC), as a common primary liver cancer, is a fatal disease worldwide (1). Since lacking specific symptoms in the early stage, the patients were always diagnosed with HCC in the advanced stage, thus losing the chance of radical treatment. In the past decade, recurrence and chemoresistance account for the unsatisfactory 5-year survival rate of HCC patients (2).

A plenty of studies have focused their attention on a distinct cell subpopulation called cancer stem cells (CSCs) in HCC which exhibited extended self-renewal potential, tumor initiating ability and resistance to chemotherapy. Previous studies showed that liver cancer stem cells could be identified by CD24, CD133, EpCAM, and other biomarkers. Numerous studies demonstrated that chemoresistance and recurrence of 
HCC were closely associated with the existence of liver CSCs. However, the detailed regulatory mechanism for generation and expansion of liver CSCs remains far from fully understood.

Belonging to RNA molecules, Long non-coding RNA (IncRNA) with more than 200 nt in length doesn't have protein encoding function. In recent years, it was found playing an important role in tumor progression, recurrence, metastasis and drug resistance (3). The inference that IncRNAs could act as oncogenes or tumor suppressors in human tumors on special conditions was confirmed by various evidence (4). It was also reported that IncRNAs could affect CSCs. For instance, THOR was upregulated in liver CSCs, then promoting HCC cells dedifferentiation and liver CSCs expansion by targeting beta-catenin signaling (5). It was found that the expression of IncRNA n339260 is associated with CSCs phenotype in HCC, and wn339260 level correlated with VM, metastasis, and shorter survival time in an animal model (6). T-cell leukemia/lymphoma 6 (TCL6), also termed TNG1 or TNG2, was a IncRNA which was not studied much. It was identified within the breakpoint cluster region on chromosome 14q32 in T-cell leukemia (7). Recent studies outlined that for patients diagnosed with clear cell renal cell carcinoma, TCL6 was related to poor prognosis (8), to early abortion (9), or to preeclampsia progression (10). What's more, previous research findings reveal a novel mechanism by which TCL6 directly binds to miR-106a-5p to suppress the proliferation, migration, and invasion via PTEN/PI3K/AKT signaling pathway in HCC. However, the functional role of TCL6 in liver CSCs remains unknown and need further exploration.

\section{Materials And Methods}

\section{Bioinformatics analyses and shRNA design}

StarBase (http://starbase.sysu.edu.cn) was utilized to analyze the correlations of IncRNA-TCL6 with cancer stem cell genes SOX11 in HCC. NCBI (https://www.ncbi.nlm.nih.gov) was used for the sequence of TCL6 and RNAi designer (http://RNAidesigner.thermofisher.com) was applied to design the RNAi of TCL6.

\section{Cell culture}

Human HCC cell lines, HepG2, MHCC-97H, and Hep3B were purchased from the China Center for Type Culture Collection (Wuhan, China). These cell lines were cultured in Dulbecco's Modifed Eagle's Medium (DMEM; Hyclone, USA, cat NO SH30022.01) supplemented with 10\% fetal bovine serum (10099141C), 100 $\mathrm{U} / \mathrm{ml}$ penicillin and $100 \mathrm{ng} / \mathrm{ml}$ streptomycin in a humidified atmosphere of $5 \%(\mathrm{v} / \mathrm{v}) \mathrm{CO}_{2}$ at $37^{\circ} \mathrm{C}$.

\section{Establishment of stable cell lines}

HEK293T cells were transfected with psPAX2, pMD2g, and PLKO-TRC-shRNA1/ shRNA2/shRNA3 or plvxmCMV-TCL6-ZsGreen1-puro with the mass ratio of 2:2:3, after $48 \mathrm{~h}$ transfection, the supernatant was collected, then centrifuged at $1000 \mathrm{rpm} / \mathrm{min}$ for $5 \mathrm{~min}$ to remove cell debris, and the supernatant was filtered through a $0.45 \mathrm{um}$ filter, measured virus titer by gradient dilutionstored and then stored at $-80^{\circ} \mathrm{C}$. The virus supernatant was infected with HepG2, MHCC-97H, and Hep3B (MOI=10). After 48 hours, the medium was replaced by completement medium and puro (puromycin $1.5 \mathrm{ug} / \mathrm{ml}$ ) was added after $96 \mathrm{~h}$. 
The screening medium was replaced every 2-3 days to remove dead cells, we observe the growth ratio of living cells or the fluorescence intensity, and express the green fluorescence by living cells to evaluate the infection efficiency, then the stably expressed cell lines were constructed from 3-4 drug screens.

\section{Flow cytometry (FCM)}

The expression proportion of CD133+ phenotype cell subsets in liver CSCs was analyzed by FACS using a Moflo XDP (Beckman Coulter, USA). The cells in logarithmic growth phase were collected for cell counting and we centrifuge the cells at room temperature for $5 \mathrm{~min}$ and discard the supernatant. The cells were washed twice with PBS, following treatment with FcR-blocking reagent (Miltenyi Biotec, Germany, Order NO 130-059-901) and then the suitable concentration cells were divided into two equal parts, respectively moved to the flow tube, incubated at $4^{\circ} \mathrm{C}$ in the dark for 15 min with CD133-PE antibody or APC-labeled control antibody, then the labeled cells were centrifuged at $1000 \mathrm{rpm} / \mathrm{min}$ for $5 \mathrm{~min}$ to discard the supernatant, resuspend with PBS, then the proportion of CD133+ phenotype cell subpopulations was measured on the machine.

\section{Spheroid formation assay}

When the cells are covered $90 \%$, then we digested with 0.25\% trypsin (Sigma, St. Louis, MO, USA, SM-2003 MSDS) washed twice with calcium/magnesium-free PBS before digesting, seeded in low-attachment 96well plates (100 cells/well) corning, USA) culturing in DMEM-F12 medium supplemented with $100 \mathrm{U} / \mathrm{ml}$ penicillin and $100 \mathrm{ng} / \mathrm{ml}$ streptomycin, $20 \mathrm{ng} / \mathrm{ml} \mathrm{EGF,} 10 \mathrm{ng} / \mathrm{ml} \mathrm{bFGF}$ and $2 \%$ B27. The cells were cultivated for 7-14 days, and the spheres were then calculated under a microscope.

\section{NA extraction and quantitative real-time polymerase chain reaction (qRT-PCR) assay}

In accordance with the manufacturer's instructions, total RNA was isolated from the liver cells using the TRIzol Total RNA Reagent (Invitrogen, USA). cDNA synthesis was performed with the above total cellular RNA as a template using the UElris II RT-PCR System for First-Strand cDNA Synthesis (US EVERBRIGHT INC, USA). 2× SYBR Green qPCR Master Mix (US EVERBRIGHT INC, USA) on an ABI 7900 system (Applied Biosystems, Foster City, CA, USA) was employed to conduct qRT-PCR assay. The glyceraldehyde 3phosphate dehydrogenase (GAPDH, AC002) was used as an internal reference. Comparative quantification was determined using the $2^{-\Delta \Delta C t}$ method. The primers were obtained from Sangon Biotech (Shanghai, China), and the sequences were summarized in Table 2.

\section{Western blot assay}

Total protein extracted from HCC cells were incubated in six well plates until $80 \%$ confluence with protein lysis buffer containing protease inhibitors (Beyotime, Beijing, China, P0013). Protein lysates were mixed with loading buffer after centrifuging at $12000 \mathrm{r} / \mathrm{min}$ for $15 \mathrm{~min}$ and boiled for ten min. $30 \mu \mathrm{g}$ of proteins were separated by SDS PAGE and transferred to PVDF membranes (Millipore, Billerica, MA, USA). The membranes were locked with $5 \%$ fat free milk for 1.5 hours at room temperature and incubated with the first antibody overnight at $4^{\circ} \mathrm{C}$. Then we washed the first antibody with cold TBST for three times and 
incubated with the HRP Goat-anti-Rabbit/mouse for 2 hours at room temperature. The first antibody included TP53 (AC002), CDKN1A (A1483), CD44 (A0340), KLF4 (A664), OCT4 (A7920), Nanog (A14150), SOX2 (A11501), GAPDH (AC002) and they were diluted in 1:1000 (ABclonal Technology, Wuhan, China). The bound antibodies were detected using Chemiluminescence imaging system (BIO-RAD, USA) with GAPDH used as a control. The images of the gels were scanned using Bio-Rad Gel Doc XR+ system (BioRad, Hercules, CA, USA) with GAPDH as an internal control.

\section{Dual luciferase reporter assay}

The full-length wild-type (WT) 3'UTR containing the predicted miR-106a-5p targeting site, and the mutanttype (MUT) 3'UTR of TP53 were amplified and cloned into the psi-check-2 vector to generate psi-check2TP53-WT and psi-check2-TP53-MUT constructs. Subsequently, the constructed luciferase vectors were transfected into HEK293T cells along with miR-106a-5p mimic or NC, respectively. After $48 \mathrm{~h}$ transfection, the relative luciferase activity was measured by normalizing the firefly luminescence to the Renilla luminescence using the Dual-Luciferase Reporter Assay System (Promega, Madison, WI, USA) following manufacturer's protocol.

\section{Statistical analysis}

All data were expressed as mean \pm SD. Differences between two groups were assessed by Student's t-test, while differences among multiple groups were performed with one-way analysis of variance or two-way analysis of Tukey. $P<0.05$ was considered to be statistically significant. Each experiment was conducted in triplicate.

\section{Results}

\section{Transfection efficiency of HCC cells}

To explore the potential role of IncRNAs in liver CSCs, we transfected liver stem cells with shRNA-TCL6 and TCL6, respectively. shRNA- TCL6 and plvx-TCL6-puro plasmids were constructed and stably transfected into HepG2, MHCC-97H, and Hep3B cells. The expression of IncRNA-TCL6 in shRNA- TCL6 or plvx-TCL6puro treated HCC cells were measured by qPCR. In the interference group, the expression of IncRNA-TCL6 was significantly lower than that of the negative control group in HepG2/MHCC-97H/Hep3B $(P<0.01)$. In the overexpression group, the expression of IncRNA-TCL6 was significantly elevated in plvx-TCL6puro groups compared with plvx-puro groups in HepG2/MHCC-97H/Hep3B $(P<0.05)$ (Figure 1).

\section{LncRNA-TCL6 overexpression decreased CD133+ LCSCs number in HCC cells}

To explore the role of IncRNA-TCL6 in cells, we transfected HepG2, MHCC-97H, and Hep3B cells with shRNA-TCL6 or plvx-TCL6-puro. Knockdown of IncRNA-TCL6 enhanced the CD133+ CSC ratio in HepG2/MHCC-97H/Hep3B. FCM test showed that the CD133+ cells were remarkably increased in shRNATCL6 group $(P<0.05)$. Overexpression of IncRNA-TCL6 decreased the CD133+ LCSC ratio in HepG2/MHCC- 
97H/Hep3B. FCM test showed that the CD133+ cell was remarkably decreased in plve-TCL6-puro group $(P<0.05)$ (Figure 2).

\section{LncRNA-TCL6 overexpression decreased sphere-forming ability of liver cancer stems in HCC}

To explore the role of TCL6 in sphere-forming ability regulation of liver cancer stems, HepG2, MHCC-97H, and Hep3B cells were transfected with shRNA-TCL6 or plve-TCL6-puro. In the knockdown group, IncRNATCL6 enhanced the CSCs self-renewal. Spheroid formation assay showed that the self-renewal capacity in shRNA-TCL6 group was remarkably enhanced in HepG2/MHCC-97H/Hep3B $(P<0.05)$; Overexpression of IncRNA-TCL6 inhibited the CSCs self-renewal. Spheroid formation assay showed that the self-renewal capacity in plvx-TCL6-puro group was remarkably reduced in HepG2 /MHCC-97H /Hep3B $(P<0.05)$ (Figure 3).

\section{Knockdown of IncRNA-TCL6 upregulated KLF4 mRNA expression and suppressed the TP53 and CDKN1A mRNA expression}

It was reported that the stem cells associated with cell cycle factors, including TP53 and P21, could have regulation function in the liver CSCs expansion (11). Meanwhile, they play a vital role in the regulation of KLF4 expression (12). Knockdown of IncRNA-TCL6 upregulated KLF4 mRNA expression and suppressed the TP53 and CDKN1A mRNA expression in HepG2/MHCC-97H/Hep3B $(P<0.05)$; Overexpression of IncRNA-TCL6 suppressed KLF4 mRNA expression and upregulated the TP53 and CDKN1A mRNA expression in HepG2/MHCC-97H/Hep3B $(P<0.05)$ (Figure 4).

\section{TCL6 overexpression modulated TP53-P21 pathway and affect the pluripotency stem cell transcriptional factors}

It was reported SOX11 regulates apoptosis of hepatocellular carcinoma by cell cycle(13). The expression levels of IncRNA-TCL6 and cancer stem cell genes SOX11 were analyzed in HCC tissue samples from satarbase dataset. The expression of InCRNA-TCL 6 was positively correlated with SOX11 expression. ***, $P<0.001$. (Table 1) In addition, literature research shows that that the stem cells associated with transcription factors, including OCT4, SOX-2, Nanog, and KLF4, could regulated the liver CSCs expansion (14-16). To further explore the possible mechanism of TCL6 regulating stem cells reprogramming in HCC, we predicted that TCL6 regulated the protein expression of the TP53-P21 pathway and pluripotency stem cell transcriptional factors. Western blot assays were performed to determine the protein levels of TP53, P21, CD44, KLF4, OCT4, Nanog, and SOX2 in HCC cells (HepG2, MHCC-97H, and Hep3B) transfected with shRNA-TCL6, plvx-TCL6-puro or matched controls (NC/plvx-puro). The obtained results suggested that HCC cells with shRNA-TCL6 showed a promotion in the protein level of CD44, KLF4, OCT4, Nanog, and SOX2, and an inhibition in the protein level of TP53 and P21, while cells with plvX-TCL6puro exerted an opposite effect on their levels (Figure 5). Taken together, these results indicated that TCL6 overexpression modulated TP53-P21 pathway and affect the pluripotency stem cell transcriptional factors in HCC cells. 
Through bioinformatics analysis, we found that TP53 contains binding sites of miR-106a-5p. Luciferase reporter assay was carried out to explore the role of TP53 in miR-106a-5p. In the assay, the luciferase reporter construct containing TP53-WT or TP53-MUT 3'UTR was established. Results revealed that miR106a-5p obviously inhibited the luciferase activity in HEK293T cells transfected with TP53 WT 3'UTR $(\boldsymbol{P}<0.01)$, but in those transfected with MUT 3'UTR, the luciferase activity wasn't inhibited significantly. Furthermore, in HCC cells transfected with miR-106a-5p inhibitor ( $\boldsymbol{P}<0.01)$, TP53 expression at mRNA level was detected evidently upregulated, while it was downregulated after $\mathrm{HCC}$ cells transfected with mimic $(\boldsymbol{P}<0.01)$. These results indicated that TP53 is a target of miR-106a-5p (Figure 6).

\section{Discussion}

$\mathrm{HCC}$, one of the most common malignant tumors, has poor prognosis in patients due to the high recurrence rate and metastasis of tumor (17). In recent years, a large amount of research reports about IncRNAs have prompted us to better understand the important role of IncRNAs in tumorigenesis.

Currently, increasing evidence pointed out that IncRNAs played a part in various biological processes such as cell growth, anti-apoptosis, migration, and invasion (18-21). Some IncRNAs have been found to be associated with stem cell properties (22). At present, the research on the mechanism of TP53/p21 pathway involved in tumor cell reprogramming is relatively mature. Research reported that reprogramming factors can activate the p53 pathway, and inhibiting the P53-p21 pathway can improve the efficiency of reprogramming $(23,24)$. However, the CSCs-related IncRNAs have rarely been reported to date and the specific mechanism of action of InCRNA-TCL6 in HCC is unclear.

LncRNA-TCL6 was first found in T-cell leukemia, located 7kb upstream of the TML1 site on chromosome $14 q 32.13(7,25)$. The TCL6 gene expressed at least 11 isoforms through very complex alternative-splicing, including splicing with the TML1 gene. Our previous research showed that TCL6 is a tumor-suppressive IncRNA and regulates PTEN/PI3K/AKT signaling pathway via directly binding to miR-106a-5p in HCC (26). Besides, TCL6 controls the process of stem cells reprogramming by interacting with pluripotent stem cell transcription factors, thus improving the prognosis of tumor patients (8). At present, some studies have shown the close association between CSCs and the reprogramming process of tumorigenesis $(27,28)$. Furthermore, the ability of self-renewal and differentiation of CSCs could determine the tumor formation and recurrence (29).

MiR-106a, which has been well studied, can be recognized as a tumor suppressor or an oncogene in various cancers. In HCC, upregulating promoter hypomethylation of miR-106a can accelerate the process of carcinogenesis(13). In addition, Literature research results showed that knockdown of miR-106a in glioma stem cells (GSC) increases the abundance of endogenous TIMP-2 protein, thereby inhibiting GSC invasion (30). Our research by luciferase reporter assay verified TP53 was a target of miR-106a-5p. Consistent with previous findings, it was tempting to speculate that TCL6 and TP53 share the same miR106a-5p binding sites, then TCL6 may up-regulate the expression of Tp53 by acting on miR-106a-5p, thereby inhibiting the stemness of liver cancer cells. 
In this paper, we studied and explored the mechanism of InCRNA-TCL6 in HCC. We analyzed the expression level of IncRNA-TCL6 in HCC by qRT-PCR. Our results indicated that the expression level of IncRNA-TCL6 was significantly lower in the interference group than the negative control group in the HepG2/MHCC-97H/Hep3B cell lines. The opposite result was obtained in the overexpression group experiment. The experimental results illustrated that knockdown of IncRNA-TCL6 enhanced the expression of CD133 in HCC cells, while overexpression of IncRNA-TCL6 showed the opposite result. Moreover, we found that the knockdown of IncRNA-TCL6 liver stem cells enhanced the ability to form a ball. The selfrenewal ability of HCC cells was significantly inhibited in plvx-TCL6-puro group. In addition, we found the relationship between shRNA-TCL6, TP53, CDKN1A, and KLF4 expression levels. The results confirmed that knockdown of shRNA-TCL6 significantly up-regulated the expression of KLF4 mRNA, and down-regulated the expression of TP53 and CDKN1A. Overexpression of TCL6 inhibited the expression of KLF4 mRNA and up-regulated the expression of TP53 and CDKN1A. Significantly, we analyzed the relationship between the expression of IncRNA-TCL6 and TP53, P21, CD44, KLF4, OCT4, Nanog, and Sox2 by western blot. The results showed that the expression of InCRNA-TCL6 was positively correlated with TP53, and P21, and negatively correlated with stem cell factors CD44, KLF4, OCT4, Nanog, and Sox2. Although we found that IncRNA-TCL6 was positively correlated with the expression of TP53 and negatively correlated with the expression of miR-106a-5p, the regulatory mechanism of miR-106a-5p on the stemness of liver cancer cells deserves further study. In summary, our research on IncRNA-TCl6 is expected to provide a novel biomarker in HCC and therapeutic target for tumor clinics.

\section{Conclusion}

Our results suggested that IncRNA-TCL6 associated with reprogramming may be a novel tumor suppressor gene in HCC, which can partially increase the expression of TP53 and inhibit the self-renewal of hepatic CSCs.

\section{Declarations}

\section{Acknowledgments}

Not applicable.

\section{Funding}

This work was supported by Hubei Province Health and Family Planning Scientific Research Project (NO: WJ2019M100).

\section{Availability of data and materials}

The datasets used and/or analysed during the current study are available from the corresponding author on reasonable request.

Ethics approval and consent to participate 
Not applicable.

\section{Consent for publication}

The authors declare that they consent to public the article in World Journal of Surgical Oncology.

\section{Competing interests}

The authors declare that they have no competing interests.

\section{Authors' contributions}

LHL and YZ conceived the experiments. MJ, BL and TZ conducted the experiments. JYZ, DHX, YZ and LHL analyzed the results. All authors read and approved the final manuscript.

\section{References}

1. Khemlina G, Ikeda S, Kurzrock R. The biology of Hepatocellular carcinoma: implications for genomic and immune therapies. Molecular cancer. 2017;16(1):149.

2. Ma J, Li T, Han X, Yuan H. Knockdown of LncRNA ANRIL suppresses cell proliferation, metastasis, and invasion via regulating miR-122-5p expression in hepatocellular carcinoma. Journal of cancer research and clinical oncology. 2018;144(2):205-14.

3. Li J, Li Z, Zheng W, Li X, Wang Z, Cui Y, et al. LncRNA-ATB: An indispensable cancer-related long noncoding RNA. Cell proliferation. 2017;50(6).

4. Fernandez-Ruiz I. Atherosclerosis: A new role for IncRNAs in atherosclerosis. Nature reviews Cardiology. 2018;15(4):195.

5. Cheng Z, Lei Z, Yang P, Si A, Xiang D, Zhou J, et al. Long non-coding RNA THOR promotes liver cancer stem cells expansion via beta-catenin pathway. Gene. 2019;684:95-103.

6. Zhao X, Sun B, Liu T, Shao B, Sun R, Zhu D, et al. Long noncoding RNA n339260 promotes vasculogenic mimicry and cancer stem cell development in hepatocellular carcinoma. Cancer science. 2018;109(10):3197-208.

7. Saitou M, Sugimoto J, Hatakeyama T, Russo G, Isobe M. Identification of the TCL6 genes within the breakpoint cluster region on chromosome 14q32 in T-cell leukemia. Oncogene. 2000;19(23):2796-802.

8. Su H, Sun T, Wang H, Shi G, Zhang H, Sun F, et al. Decreased TCL6 expression is associated with poor prognosis in patients with clear cell renal cell carcinoma. Oncotarget. 2017;8(4):5789-99.

9. Liu LP, Gong YB. LncRNA-TCL6 promotes early abortion and inhibits placenta implantation via the EGFR pathway. European review for medical and pharmacological sciences. 2018;22(21):7105-12. 
10. Wu JL, Wang YG, Gao GM, Feng L, Guo N, Zhang CX. Overexpression of IncRNA TCL6 promotes preeclampsia progression by regulating PTEN. European review for medical and pharmacological sciences. 2019;23(10):4066-72.

11. Deng J, Yang M, Jiang R, An N, Wang X, Liu B. Long Non-Coding RNA HOTAIR Regulates the Proliferation, Self-Renewal Capacity, Tumor Formation and Migration of the Cancer Stem-Like Cell (CSC) Subpopulation Enriched from Breast Cancer Cells. PloS one. 2017;12(1):e0170860.

12. Anuja K, Chowdhury AR, Saha A, Roy S, Rath AK, Kar M, et al. Radiation-induced DNA damage response and resistance in colorectal cancer stem-like cells. International journal of radiation biology. 2019;95(6):667-79.

13. Yuan R, Zhi Q, Zhao H, Han Y, Gao L, Wang B, et al. Upregulated expression of miR-106a by DNA hypomethylation plays an oncogenic role in hepatocellular carcinoma. Tumour biology : the journal of the International Society for Oncodevelopmental Biology and Medicine. 2015;36(4):3093-100.

14. Abulaiti X, Zhang H, Wang A, Li N, Li Y, Wang C, et al. Phosphorylation of Threonine(343) Is Crucial for OCT 4 Interaction with SOX2 in the Maintenance of Mouse Embryonic Stem Cell Pluripotency. Stem cell reports. 2017;9(5):1630-41.

15. van Schaijik B, Davis PF, Wickremesekera AC, Tan ST, Itinteang T. Subcellular localisation of the stem cell markers OCT4, SOX2, NANOG, KLF4 and c-MYC in cancer: a review. Journal of clinical pathology. 2018;71(1):88-91.

16. Loh YH, Wu Q, Chew JL, Vega VB, Zhang W, Chen X, et al. The Oct4 and Nanog transcription network regulates pluripotency in mouse embryonic stem cells. Nature genetics. 2006;38(4):431-40.

17. Ni W, Zhang Y, Zhan Z, Ye F, Liang Y, Huang J, et al. A novel IncRNA uc.134 represses hepatocellular carcinoma progression by inhibiting CUL4A-mediated ubiquitination of LATS1. Journal of hematology \& oncology. 2017;10(1):91.

18. Zhao W, Geng D, Li S, Chen Z, Sun M. LncRNA HOTAIR influences cell growth, migration, invasion, and apoptosis via the miR-20a-5p/HMGA2 axis in breast cancer. Cancer medicine. 2018;7(3):842-55.

19. Liu Y, Yang Y, Li L, Liu Y, Geng P, Li G, et al. LncRNA SNHG1 enhances cell proliferation, migration, and invasion in cervical cancer. Biochemistry and cell biology = Biochimie et biologie cellulaire. 2018;96(1):38-43.

20. Sha L, Huang L, Luo X, Bao J, Gao L, Pan Q, et al. Long non-coding RNA LINC00261 inhibits cell growth and migration in endometriosis. The journal of obstetrics and gynaecology research. 2017;43(10):1563-9.

21. Zhao L, Han T, Li Y, Sun J, Zhang S, Liu Y, et al. The IncRNA SNHG5/miR-32 axis regulates gastric cancer cell proliferation and migration by targeting KLF4. FASEB journal : official publication of the 
Federation of American Societies for Experimental Biology. 2017;31(3):893-903.

22. Fu Z, Chen C, Zhou Q, Wang Y, Zhao Y, Zhao X, et al. LncRNA HOTTIP modulates cancer stem cell properties in human pancreatic cancer by regulating HOXA9. Cancer letters. 2017;410:68-81.

23. Kawamura T, Suzuki J, Wang YV, Menendez S, Morera LB, Raya A, et al. Linking the p53 tumour suppressor pathway to somatic cell reprogramming. Nature. 2009;460(7259):1140-4.

24. Li W, Tian E, Chen ZX, Sun G, Ye P, Yang S, et al. Identification of Oct4-activating compounds that enhance reprogramming efficiency. Proceedings of the National Academy of Sciences of the United States of America. 2012;109(51):20853-8.

25. Chen Z, Zhuang Q, Cheng K, Ming Y, Zhao Y, Ye Q, et al. Long non-coding RNA TCL6 enhances preferential toxicity of paclitaxel to renal cell carcinoma cells. Journal of Cancer. 2020;11(6):1383-92.

26. Luo LH, Jin M, Wang LQ, Xu GJ, Lin ZY, Yu DD, et al. Long noncoding RNA TCL6 binds to miR-106a$5 p$ to regulate hepatocellular carcinoma cells through PI3K/AKT signaling pathway. Journal of cellular physiology. 2020;235(9):6154-66.

27. Shen YA, Wang CY, Hsieh YT, Chen YJ, Wei YH. Metabolic reprogramming orchestrates cancer stem cell properties in nasopharyngeal carcinoma. Cell cycle (Georgetown, Tex). 2015;14(1):86-98.

28. Friedmann-Morvinski D, Verma IM. Dedifferentiation and reprogramming: origins of cancer stem cells. EMBO reports. 2014;15(3):244-53.

29. Bao B, Ahmad A, Azmi AS, Ali S, Sarkar FH. Overview of cancer stem cells (CSCs) and mechanisms of their regulation: implications for cancer therapy. Current protocols in pharmacology. 2013;Chapter 14:Unit 14.25.

30. Wang Z, Wang B, Shi Y, Xu C, Xiao HL, Ma LN, et al. Oncogenic miR-20a and miR-106a enhance the invasiveness of human glioma stem cells by directly targeting TIMP-2. Oncogene. 2015;34(11):1407-19.

\section{Tables}

TABLE 1 The Correlations of TCL6 with cancer stem cell genes SOX11 in liver hepatocellular carcinoma (LIHC).

$\begin{array}{ll}\text { Gene name } & \text { Correlation coefficients with TCL6 } \\ \text { S0X11 } & R=0.16, P<0.0001\end{array}$

TABLE 2 Sequence information of primers

\section{Figures}




\section{Primer Sequence $5^{\prime} \mathbf{3}^{\prime}$}

TCL6- CCGGAAAAGCAGACAACCACACAAACTCGAGTTTGTGTGGTTGTCTGCTTTTTTTTTGGTACC shRNAforward

TCL6ShRNAreverse

shRNA CCGGCCTAAGGTTAAGTCGCCCTCGCTCGAGCGAGGGCGACTTAACCTTAGGTTTTTGGTACC controlforward

shRNA AATTGGTACCAAAAACCTAAGGTTAAGTCGCCCTCGCTCGAGCGAGGGCGACTTAACCTTAGG controlreverse

qPCR- $\quad$ ACATCTGGGCCCCCTGGAACGCTTCAGTCATA

TCL6forward

qPCR- CAGATTCGAGTCACGTCCT

TCL6-

reverse

qPCR- ACGGATTTGGTCGTATTGGGCG

GAPDH-

forward

qPCR- GCTCCTGGAAGATGGTGATGGG

GAPDH-

reverse

qPCR- ACCTATGGAAACTACTTCCTGAAA

P53-

forward

qPCR- CTGGCATTCTGGGAGCTTCA

P53-

reverse

qPCR- CCCGTGAGCGATGGAACTT

P21-

forward

qPCR- GCGTTTGGAGTGGTAGAAATCT

P21-

reverse

QPCR- CCCAATTACCCATCCTTCCT

KLF4-

forward

qPCR- AGGTTTCTCACCTGTGTGGG

KLF4-

reverse 
a

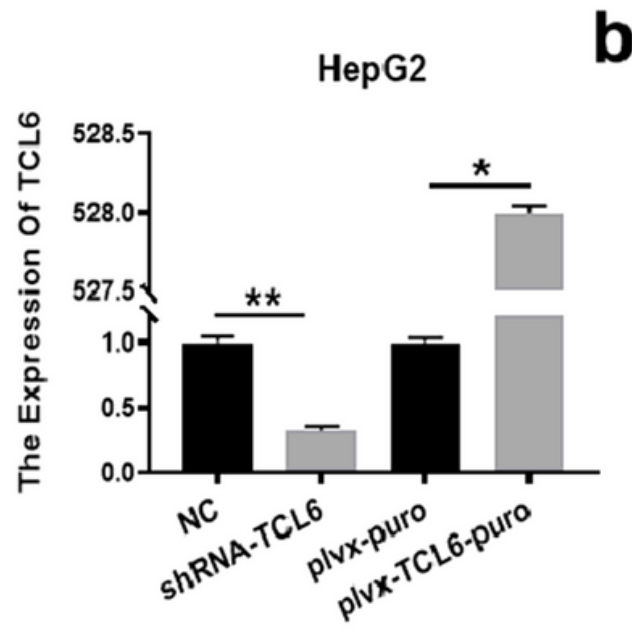

b

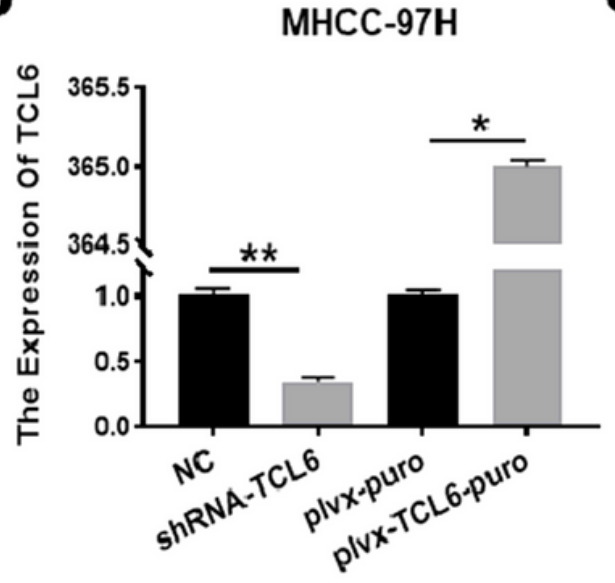

C

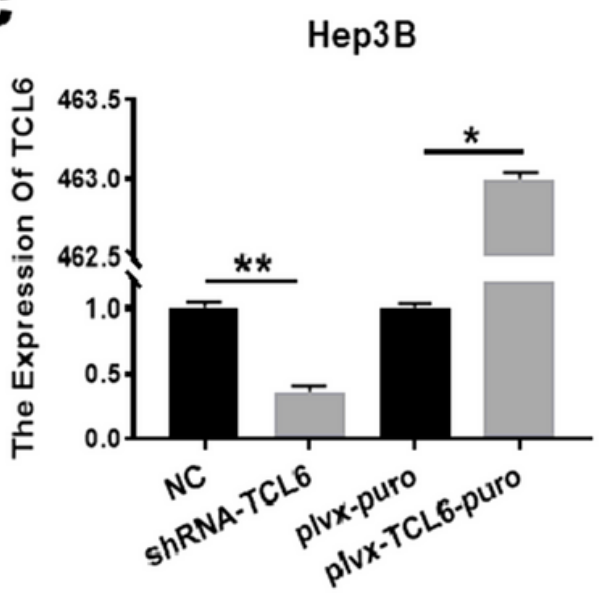

Figure 1

The efficiency of overexpression or knockdown of TCL6 was verified by qRT-PCR in HCC cells. $(a, b$ and $c)$ The expression of TCL6 was decreased in shRNA-TCL6 groups compared with NC groups and was apparently overexpressed in cells transfected with plvx-TCL6-puro compared with plvx-puro groups in HepG2/ MHCC-97H/Hep3B. *, $\mathrm{P}<0.05$; **, $\mathrm{P}<0.01$. Differences between two groups were assessed by Student's t-test. $\mathrm{P}<0.05$ was considered to be statistically significant. Each experiment was conducted in triplicate. 
a

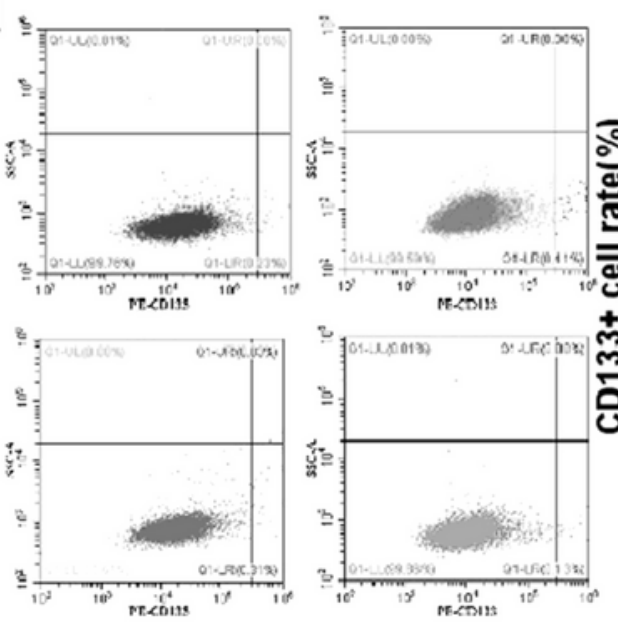

b
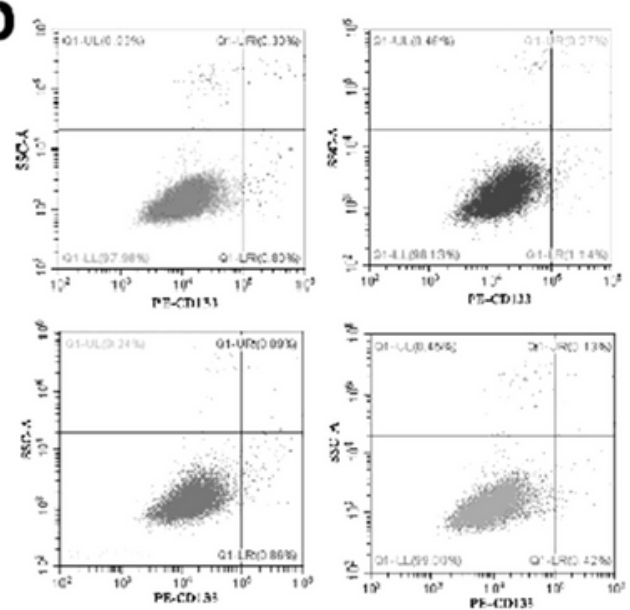

C
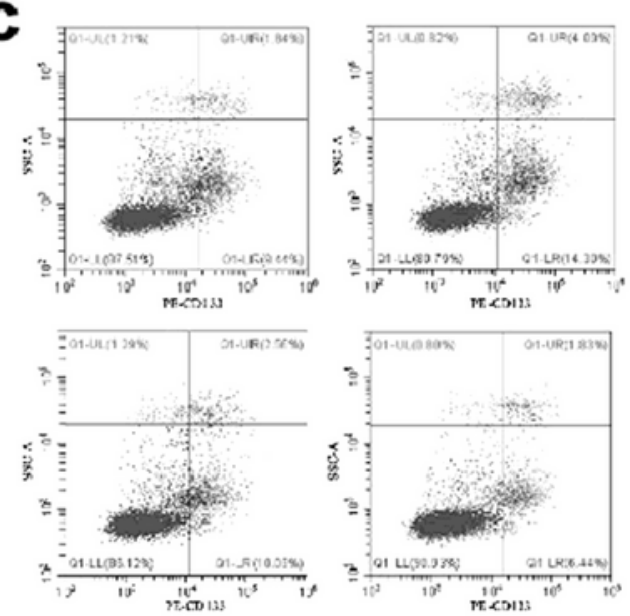

HepG2

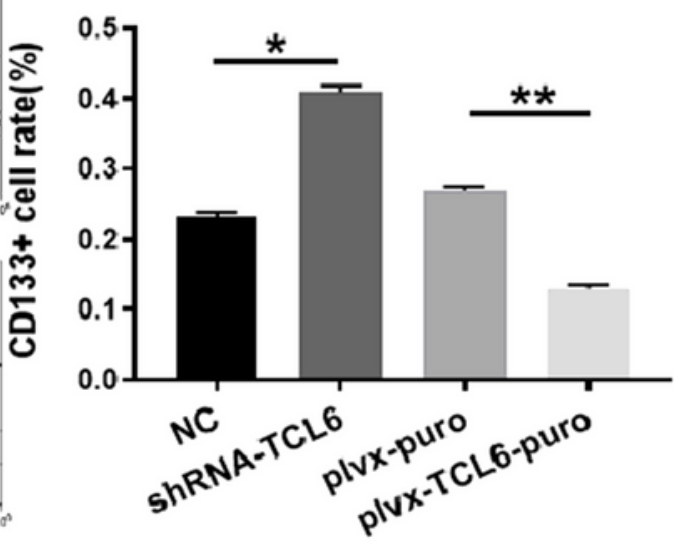

MHCC-97H

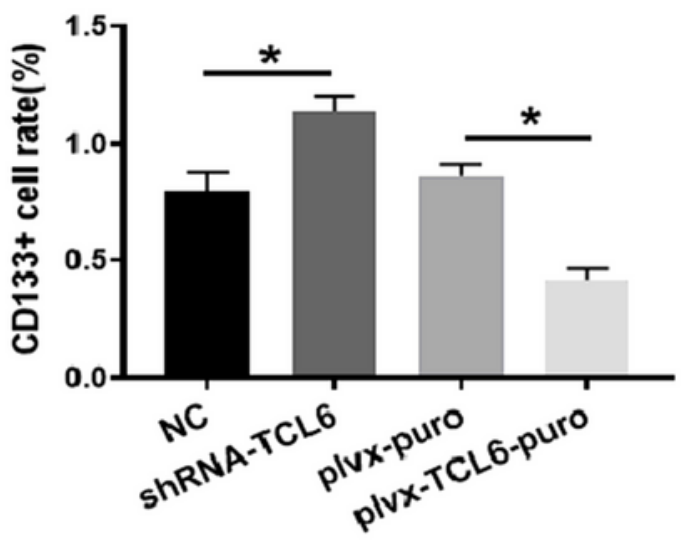

Hep3B

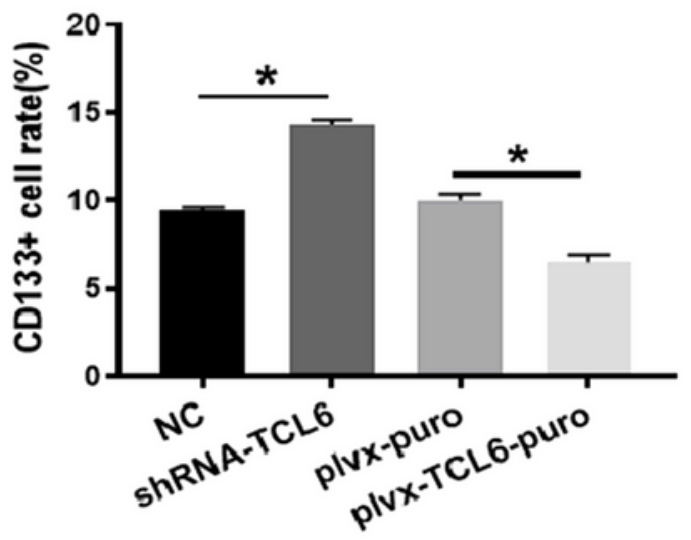

Figure 2

Knockdown of IncRNA-TCL6 enhanced the CD133+ CSC ratio in HCC cells. ( $a$, b and c) FCM test showed that the CD133+ cells were remarkably increased in shRNA-TCL6 group; overexpressed of IncRNA-TCL6 reduced the CD133+ CSC ratio in HepG2/ MHCC-97H/Hep3B. FCM test showed that the CD133+ cells were remarkably decreased in plvx-TCL6-puro group, *, $\mathrm{P}<0.05$; **, $\mathrm{P}<0.01$. 
a

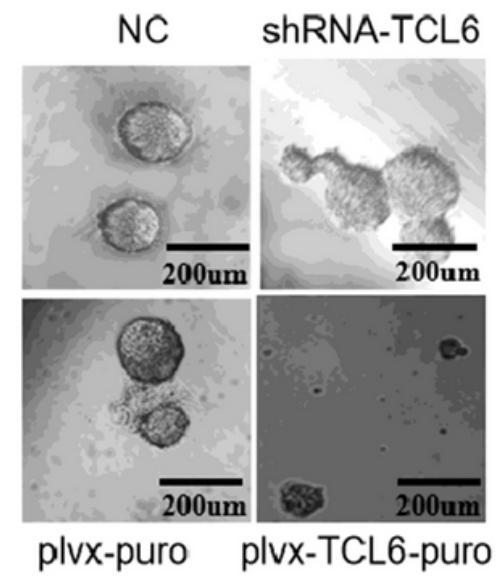

b

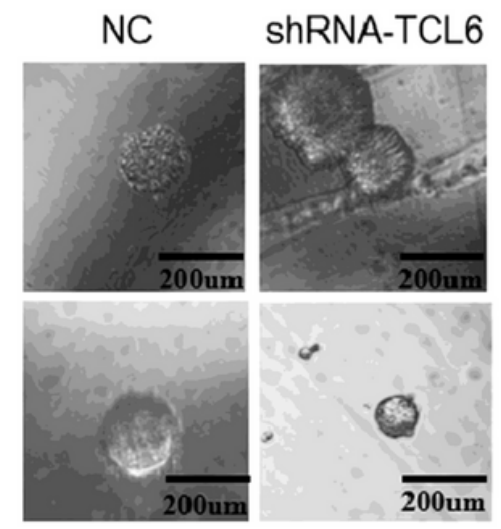

plvx-puro plvx-TCL6-puro

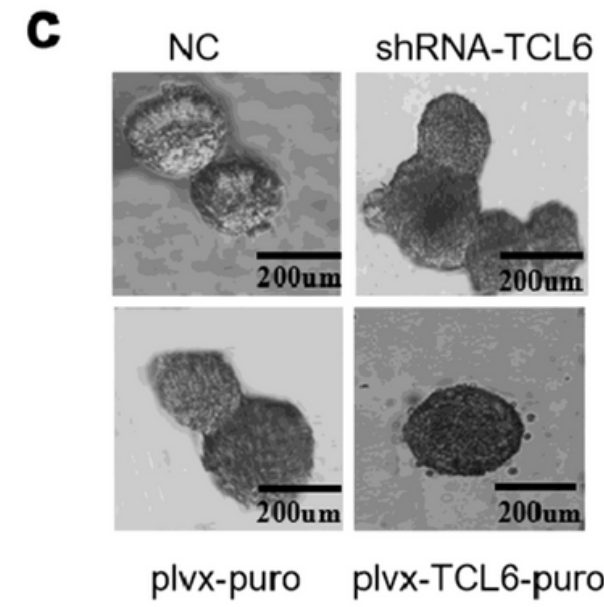

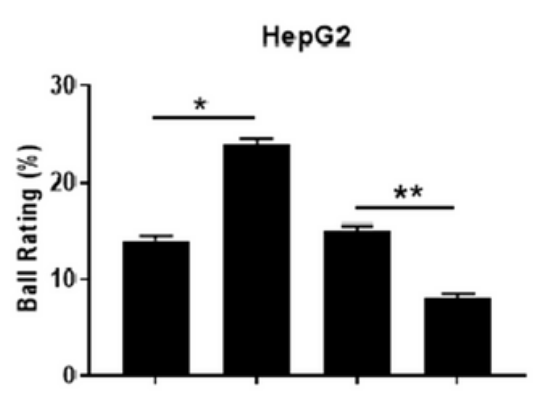

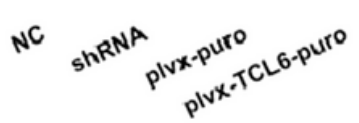

MHCC-97H

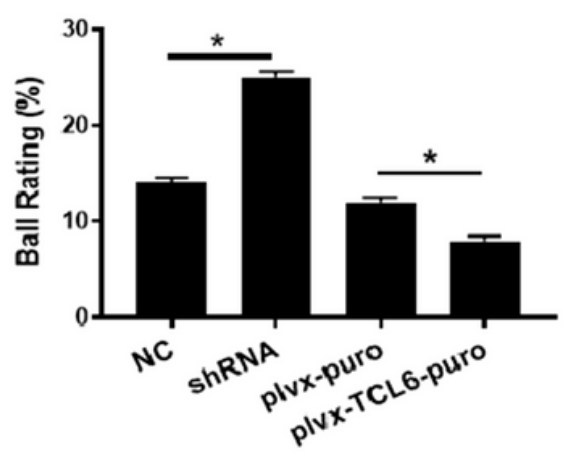

Hep3B

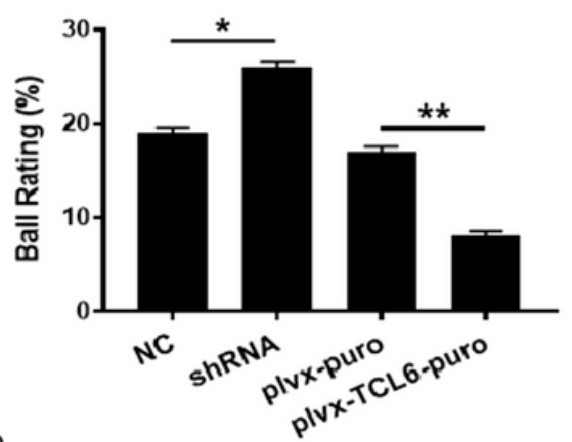

HepG2

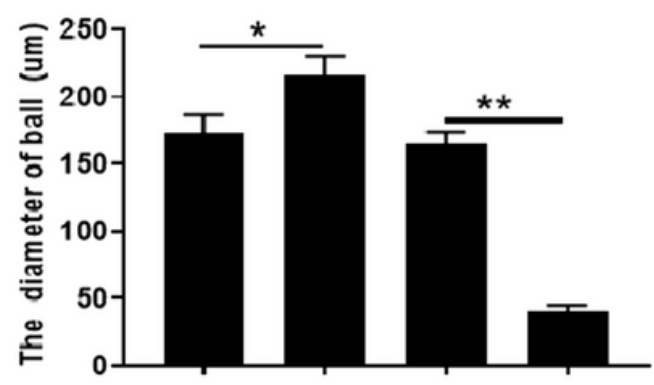

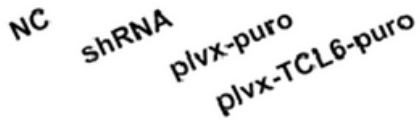

MHCC-97H

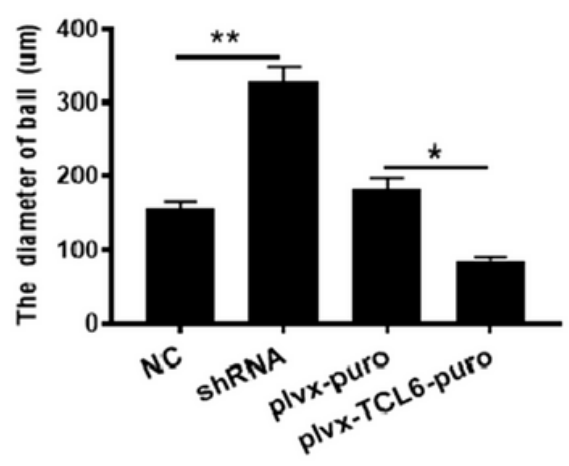

Нер3B

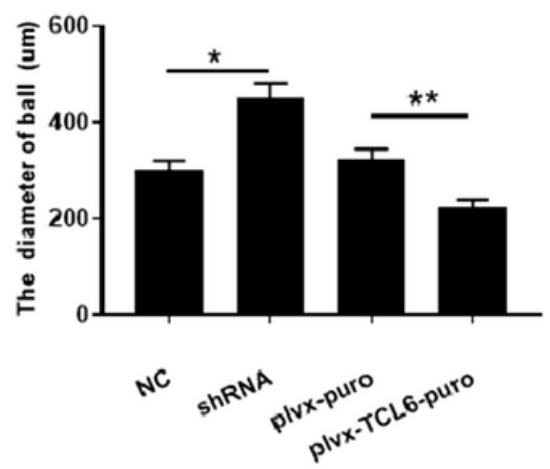

Figure 3

Knockdown of IncRNA TCL6 enhanced the CSC self-renewal in HCC cells. (a, b and c) Spheroid formation assay showed that the self-renewal capacity in shRNA-TCL6 group was remarkably enhanced; overexpressed IncRNA-TCL6 reduced the CSCs self-renewal in HepG2/MHCC-97H/Hep3B. Spheroid formation assay showed that the self-renewal capacity in plvx-TCL6-puro group was remarkably reduced in HepG2/MHCC-97H/Hep3B. * $\mathrm{P}<0.05$; **, $\mathrm{P}<0.01$. Differences among multiple groups were performed with one-way analysis of variance. $\mathrm{P}<0.05$ was considered to be statistically significant. Each experiment was conducted in triplicate. Each well plate was planted with 100 cells, and the average forball rating (The 
forball rating was calculated based on the formula of number of balls/100) of each group was calculated under the microscope after 14-day cultivation, the difference in forball ability between each group was compared based on the ball rate and diameter of the ball. Meanwhile, a representative field of view was selected to show forball ability intuitively.
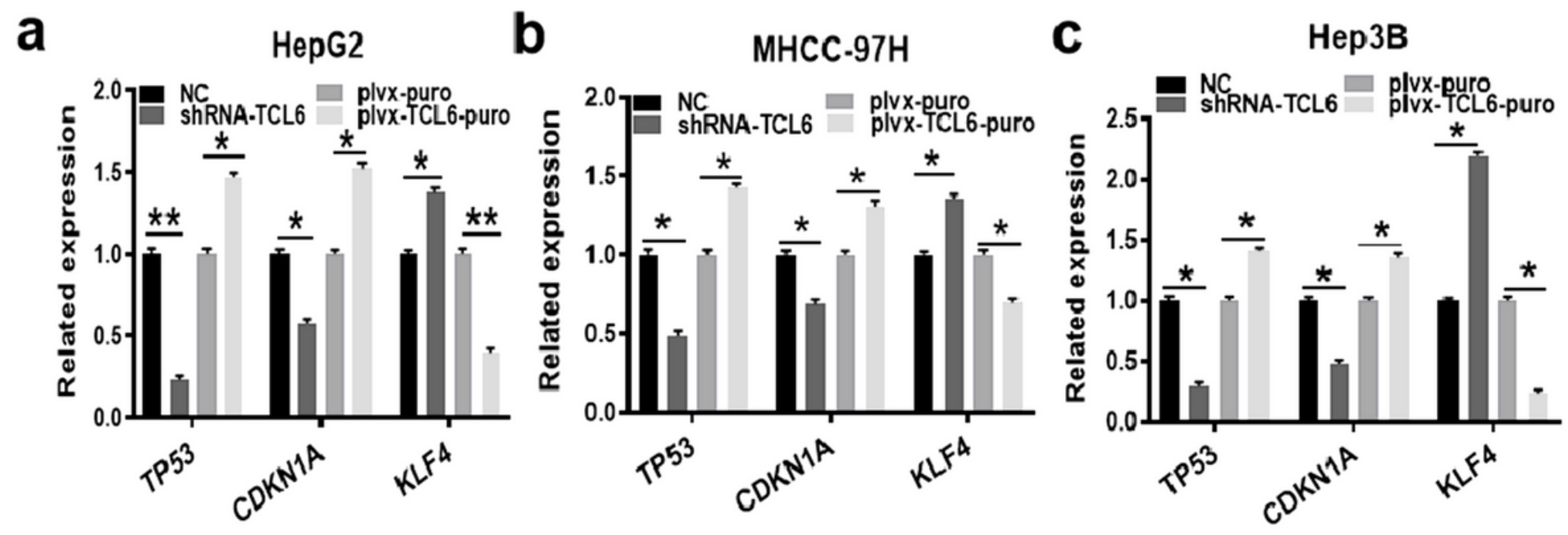

\section{Figure 4}

Expression of TP53, CDKN1A and cancer stem cell gene KLF4 were measured by qPCR in steady cells. (a, $\mathrm{b}$ and c) Expression of TP53 and CDKN1A was significantly reduced in shRNA-TCL6 groups compared with NC groups in HepG2, while the KLF4 expression was remarkably elevated; Expression of TP53 and CDKN1A was significantly enhanced in plvx-TCL6-puro groups compared with plvx-TCL6-puro groups in HepG2/MHCC-97H/Hep3B ( $\left.{ }^{*}, \mathrm{P}<0.05\right)$, while the KLF4 expression was remarkably decreased. ${ }^{*}, \mathrm{P}<0.05$; **, $\mathrm{P}<0.01$. Differences among multiple groups were performed with two-way analysis of Tukey. $\mathrm{P}<0.05$ was considered to be statistically significant. Each experiment was conducted in triplicate. 
a

NC

$\begin{array}{lllll}\operatorname{shRNA-TCL6} & - & - & - & + \\ \text { plvx-puro } & + & - & - & - \\ \text { plvx-TCL6-puro } & - & + & - & -\end{array}$

TP53
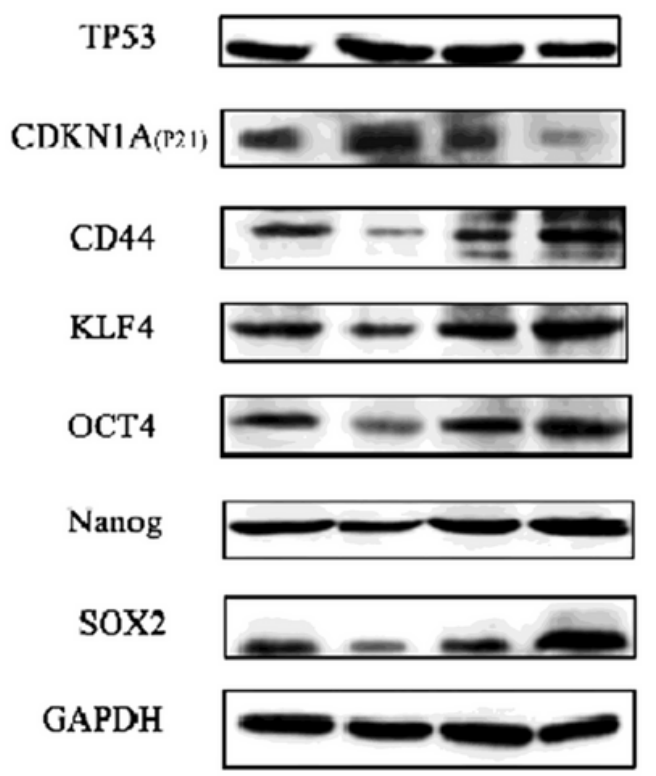

HepG2

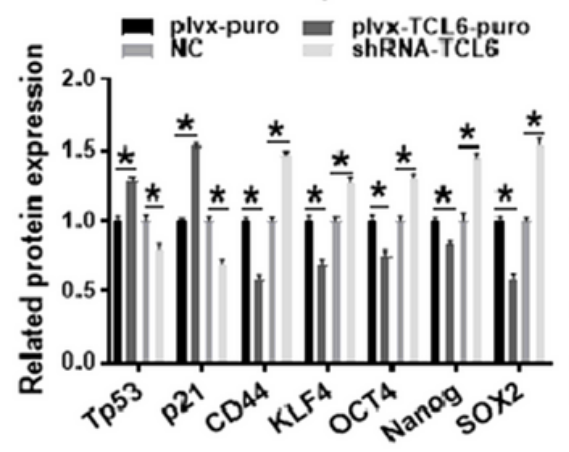

b MHCC-97H
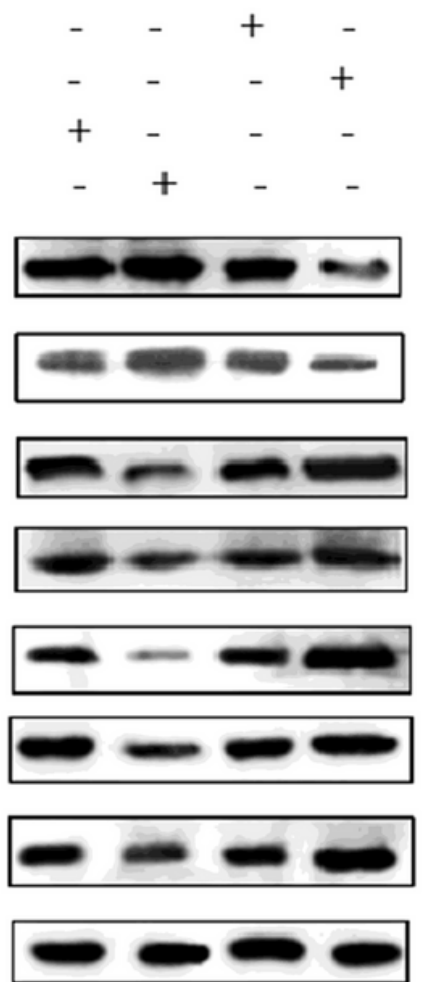

MHCC-97H

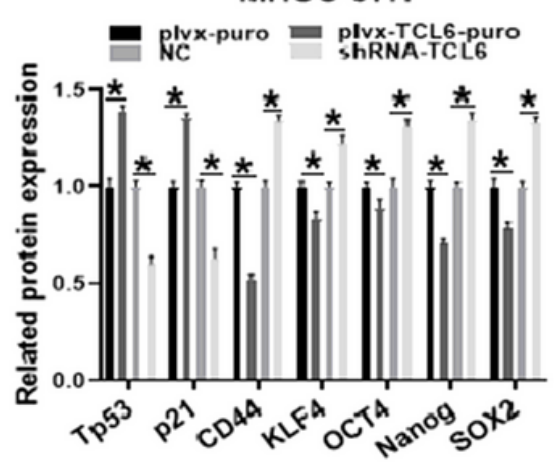

C

Hep3B

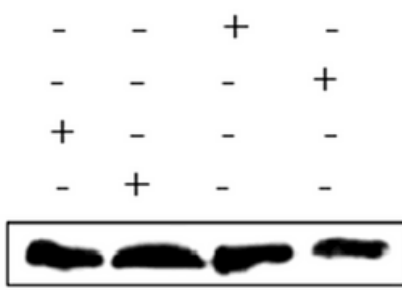

$55 \mathrm{kDa}$

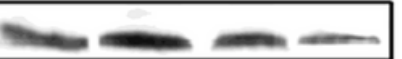

$18 \mathrm{kDa}$

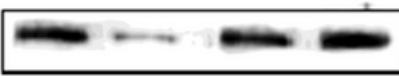

$82 \mathrm{kDa}$

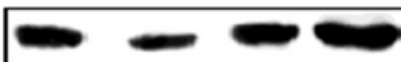

$50 \mathrm{kDa}$

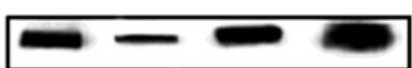

$38 \mathrm{kDa}$

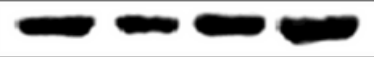

$48 \mathrm{kDa}$

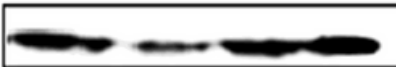

$34 \mathrm{kDa}$

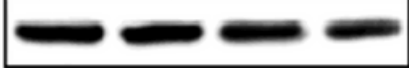

$37 \mathrm{kDa}$

\section{Hep3B}

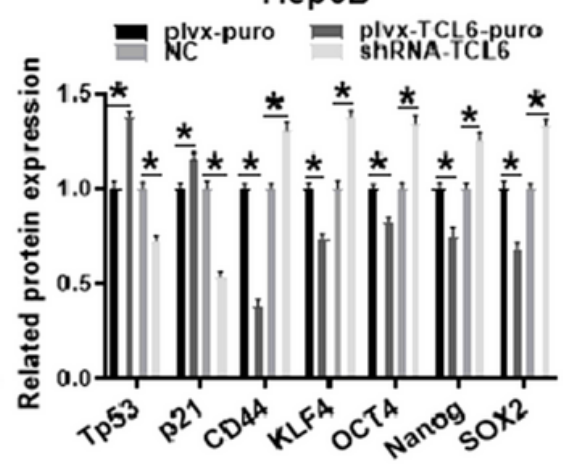

Figure 5

Protein expression of TP53, CDKN1A and cancer stem cell gene CD44, KLF4, OCT4, Nanog, and SOX2 was measured by Western blot in steady cells. ( $a, b$ and $c$ ) IncRNA-TCL6 overexpression promoted the protein expression of TP53 and CDKN1A, suppressed the protein expression of CD44, KLF4, OCT4, Nanog, and SOX2 in HepG2/ MHCC-97H/Hep3B. And downregulation of TCL6 showed the opposite result, *, P<0.05. Differences among multiple groups were performed with two-way analysis of Tukey. $\mathrm{P}<0.05$ was considered to be statistically significant. Each experiment was conducted in triplicate. 
a

TP53 WT: 3558- catacetgtaatctcAGCACTTTg-3581

miR-106a-5p: gauggacgugacauUCGUGAAAa

TP53 MUT: 3558- catacctgtaatctcAGCACTTTg-3581

C

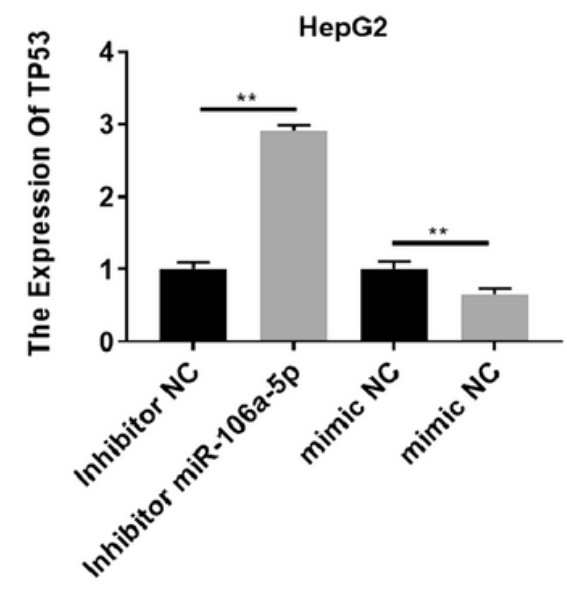

b
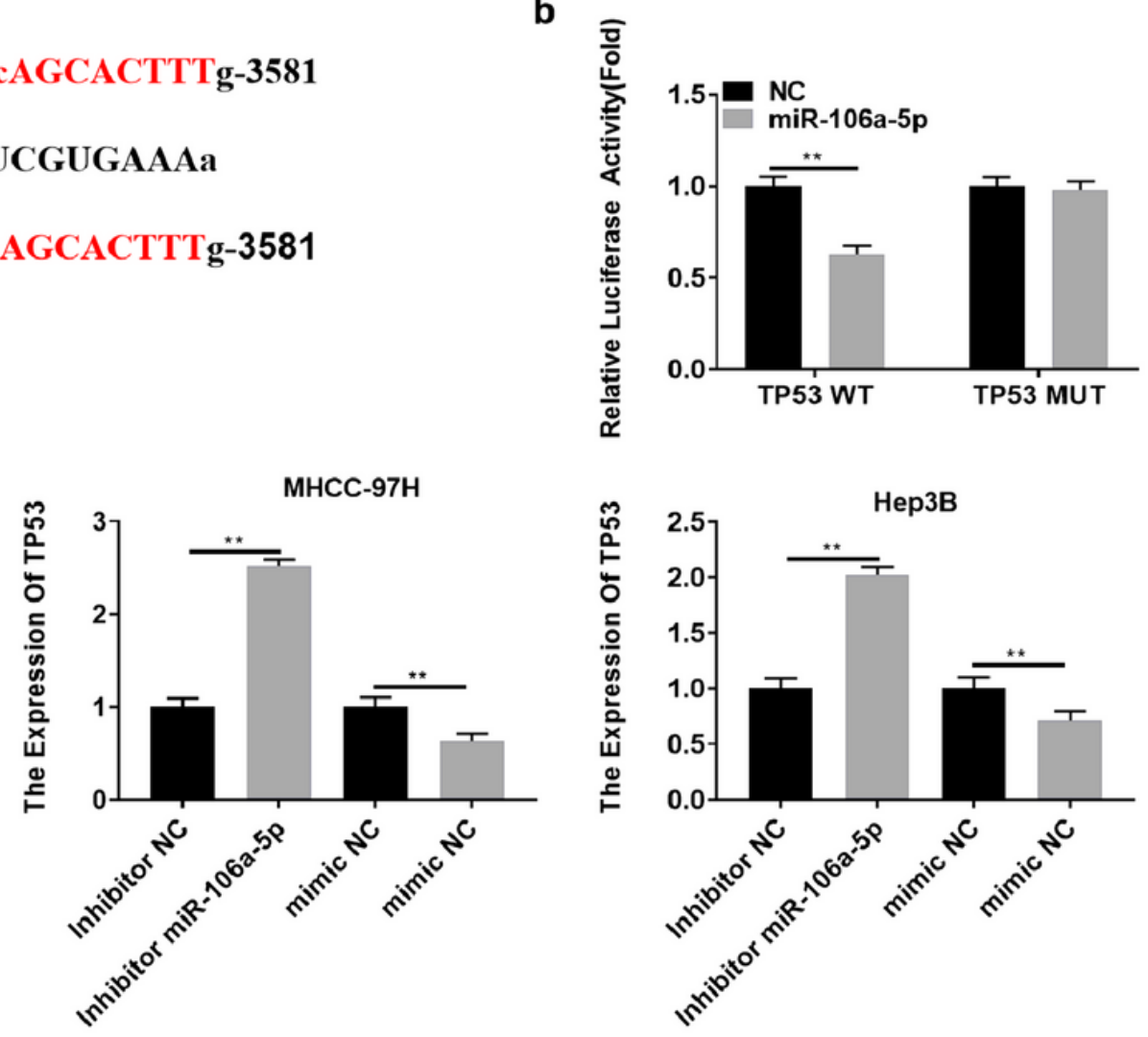

Figure 6

TCL6 regulated the expression of TP53 via competitively interacting with miR-106a-5p in HCC cells. (a) The 3'UTR of TP53 harbors one cognate site in miR-106a-5p, and TCL6 and TP53 share the same miRNA binding site based on StarBase. TP53 MUT was generated by mutation at the paired bases. (b) Effect of miR-106a-5p on the luciferase reporter activity of cells with TP53 WT or MUT 3'UTR. (c) Effects of miR106a-5p overexpression or knockdown on the expression of TP53 analyzed by qRT-PCR in HepG2, MHCC97H and Hep3B cells. **, P<0.01. TCL6: T cell leukemia/lymphoma 6; miR-106a-5p: microRNA-106a-5p; WT: wild type; MUT: mutant type. 